\title{
Does prior endoscopic cyclophotocoagulation (ECP) affect subsequent trabeculectomy outcomes?
}

\author{
Abhijit Anand Mohite ${ }^{1}$ (D) Emma Samia-Aly ${ }^{1}$ - Uthaya Shankar Ramanathan ${ }^{1}$. Patrick G. Corridan ${ }^{1}$. \\ Shashidhar Murthy ${ }^{1}$
}

Received: 7 April 2021 / Revised: 7 September 2021 / Accepted: 16 October 2021 / Published online: 25 October 2021

(c) The Author(s), under exclusive licence to Springer-Verlag GmbH Germany, part of Springer Nature 2021

\begin{abstract}
Purpose To report long-term outcomes of trabeculectomy following prior endoscopic cyclophotocoagulation (ECP). Methods Retrospective case-controlled comparative study reporting 2-year outcomes of eyes undergoing trabeculectomy following failed prior ECP (group 1), using eyes undergoing trabeculectomy as a primary glaucoma procedure as controls (group 2). Results Filtration surgery was required in only $19.4 \%$ (12/62) of eyes undergoing ECP. Of these, nine eyes that underwent trabeculectomies were included in group 1. Nine matched eyes were used as controls and included in group 2. Mean baseline IOPs were $23.7 \pm 7.7$ and $26.0 \pm 6.7 \mathrm{mmHg}(p=0.452)$ in groups 1 and 2 , respectively, on a mean of $3.4 \pm 0.9$ and $2.8 \pm 1.4$ medications, respectively $(p=0.274)$. The mean 2 -year IOP was $10.6 \pm 5.2$ and $12.9 \pm 4.0 \mathrm{mmHg}(p=0.285)$ in groups 1 and 2 , respectively, on a mean of $0.1 \pm 0.3$ and $0.1 \pm 0.4$ medications $(p=0.931)$ respectively. Complete success rates were $77.8 \%$ and $88.9 \%$ in groups 1 and 2 , respectively $(p=0.527)$. Qualified success rates were $11.1 \%$ in both groups. Combined success rates were $88.9 \%$ and $100.0 \%$ in groups 1 and 2 , respectively $(p=0.318)$. There were no failures in group 2 , compared to $11.1 \%$ in group $1(p=0.318)$. There were no significant between-group differences in hypotony, bleb needling, cystoid macular oedema, persistent uveitis, or repeat filtration surgery rates.

Conclusions ECP is a safe initial procedure in eyes with progressive glaucomas, and may substantially reduce the need for trabeculectomy without compromising outcomes in the minority that go on to require it.
\end{abstract}

\section{Key messages}

\section{What was known before}

- Trabeculectomy is still regarded as the gold-standard glaucoma filtration procedure, but is more 'labour-intensive' with regards to post-operative follow-up. Endoscopic cyclophotocoagulation (ECP) is an effective minimally invasive procedure that is both quicker and requires less frequent post-operative follow-up. However it has mainly been reserved for early glaucomas when used as a primary procedure.

\section{What this study adds}

- ECP substantially reduces the need for a trabeculectomy in uncontrolled moderate and advanced glaucomas.

- Prior ECP does not adversely affect the long-term safety or efficacy of subsequent trabeculectomy surgery if required.

- $\quad$ ECP can be considered as a safe primary glaucoma procedure in progressive glaucomas and it may help to better serve the expected surge of cases in the aftermath of the covid-19 pandemic.

Abhijit Anand Mohite and Emma Samia-Aly contributed equally to this work and are joint first authors

Abhijit Anand Mohite

abhijit.mohite2@nhs.net

1 Wolverhampton and Midland Counties Eye Infirmary, New Cross Hospital, The Royal Wolverhampton Hospitals NHS
Trust, Wolverhampton Road, Wolverhampton WV10 0QP, UK 
Keywords Trabeculectomy $\cdot$ Endoscopic cyclophotocoagulation $\cdot$ Minimally invasive glaucoma surgery $\cdot$ Glaucoma

\section{Introduction}

It is conventional practice to avoid cyclo-destructive procedures in eyes with uncontrolled glaucoma and good visual potential due to the risk of chronic inflammation, profound sight-threatening hypotony, and eventual phthisis [1]. Consequently, cyclo-ablative laser procedures have traditionally been reserved for eyes with poor visual potential, or as a last resort after multiple glaucoma filtration surgeries have failed [1]. Although there is some, albeit limited, evidence to suggest the safety and efficacy of transscleral diode laser cyclophotocoagulation for temporary intraocular pressure (IOP) control for medically refractory glaucoma prior to trabeculectomy surgery [2], general consensus has been to avoid cyclo-destructive procedures prior to a trabeculectomy.

The development of endoscopic cyclophotocoagulation (ECP) has, however, led to somewhat of a paradigm shift, with ECP being used earlier on in the management pathway thanks to the ability to specifically target and titrate treatment to the ciliary processes without causing significant collateral tissue damage [3]. Histologic studies have supported this and have demonstrated less tissue disruption using ECP compared to transscleral cyclophotocoagulation [3].

ECP was first introduced by Shields in 1968 as an alternative to the more commonly used transscleral approach, and was initially only used in aphakic eyes [4]. Unlike other minimally invasive glaucoma surgeries (MIGS) that enhance aqueous outflow pathways, ECP targets aqueous inflow and aims to lower IOP by reducing aqueous production $[5,6]$. Its use is not limited to refractory glaucomas, with several studies demonstrating efficacy in eyes with moderate-to-advanced glaucomas, either as a standalone procedure or in combination with cataract surgery [7].

Several studies have reported good long-term efficacy in terms of IOP reduction following ECP in glaucoma patients [8-10]. Despite this, its widespread uptake has been modest, with most surgeons preferring to adopt various MIGS devices developed to target aqueous outflow instead. Given the profile of inflammatory complications, there may be concerns about sequelae with respect to the survival of any future glaucoma filtration surgery. Fibrin exudation (24\%), hyphaema (12\%), cystoid macular edema (10\%), choroidal detachment (4\%), and malignant glaucoma $(1 \%)$ have been reported as relatively common complications after ECP in some studies [8]. More serious complications including hypotony (up to $8 \%$ ) and phthisis bulbi (up to 3\%) have been reported [11], but less commonly as compared to transscleral cyclophotocoagulation. A large study of 5824 eyes undergoing ECP reported low rates of choroidal effusion $(0.4 \%)$, retinal detachment
( $0.3 \%)$, hypotony/phthisis $(0.1 \%)$, and massive choroidal haemorrhage $(0.1 \%)$ over a mean follow-up of 5.2 years, with only $1 \%$ of patients experiencing a vision loss of more than two lines [12].

As is the case with other MIGS procedures, a proportion of eyes undergoing ECP still need subsequent glaucoma filtration surgery (GFS) to achieve target IOP. A number of studies report a good safety and efficacy profile of ECP in eyes with prior failed trabeculectomies or glaucoma drainage devices $[8,13]$. However, no studies to our knowledge have reported on rates of subsequent GFS after undergoing ECP as a primary procedure. Furthermore, no studies to date have investigated whether ECP adversely affects the outcomes of any subsequent GFS.

We sought to address this gap by providing real-world data on the long-term outcomes of trabeculectomy surgery in eyes that have previously undergone ECP as a primary glaucoma procedure, specifically looking at rates of chronic hypotony, cystoid macular oedema, and early bleb failure. We hypothesized that prior ECP would lead to higher rates of bleb encapsulation, bleb needling revisions, and bleb failure compared to trabeculectomies that have not undergone prior ECP.

\section{Methods}

This was a retrospective case-control comparative study in a predominantly Caucasian population. Cases (group 1) consisted of all eyes that underwent a trabeculectomy following prior ECP, whilst controls (group 2) were identified from a list of primary trabeculectomies performed within the same time period. Control eyes were matched as close as possible to the study group with regard to glaucoma severity, ethnicity, age, surgeon, IOP lowering medications, and previous laser trabeculoplasty, and had not undergone any form of prior cyclo-destructive laser procedures. All eyes fulfilling these criteria were included in this control group.

A minimum of 2-year post-operative follow-up data was required for eyes to be included in the study. All trabeculectomies were performed by two experienced glaucoma consultants at the Wolverhampton and Midland Counties Eye Infirmary, New Cross Hospital, The Royal Wolverhampton Hospitals NHS Trust, UK.

Patients were selected to undergo primary ECP if they had moderate or advanced progressive primary open-angle glaucoma (POAG), normal tension glaucoma (NTG), or secondary open-angle glaucoma, but were not ideally suited or 
agreeable for primary trabeculectomy surgery. Phakic eyes underwent combined phacoemulsification and ECP surgery (phaco-ECP). Eyes that had received previous transscleral cyclophotocoagulation or those with previous GFS, angle closure disease, and complex uveitic or end-stage glaucoma were relative contra-indications for ECP.

Pre- and post-operative IOP, visual acuity, glaucoma medications, complications, and further glaucoma interventions were recorded retrospectively from patient case notes at each time point.

Primary outcome measures were complete and qualified success rates, as well as failure rates. Success was defined as IOP $<16 \mathrm{mmHg}$ without (complete success) or with (qualified success) the need for topical glaucoma medications at 2 years after trabeculectomy. Failure was defined as the need for further GFS or loss of light perception in the 2 years after trabeculectomy. The need for bleb needling revision (BNR) was not deemed a failure if good IOP control was still achieved.

Secondary outcome measures were post-operative complications. These included clinical hypotony with hypotonyrelated complications, a return to theatre including for BNR, and severe vision threatening complications such as suprachoroidal haemorrhage or endophthalmitis.

Statistical analyses were performed using SPSS Statistics version 23.0 (IBM, New York, USA). Chi-squared or Fisher's exact tests were used for between-group comparisons of categorical variables whilst the Mann-Whitney $U$ test was used for between-group comparisons of continuous data. Kaplan-Meier analysis was performed for each group. Statistical significance was set at $p<0.05$ and, where possible, standard deviations (SD) of means were calculated. The study adhered to the tenets of the Declaration of Helsinki and all patient data extracted were anonymized for analysis. All procedures were performed following informed patient consent, and the project was registered as an audit within the local department.

\section{ECP technique}

All ECP procedures were performed under subtenon or peribulbar local anaesthesia using a standard technique. This consisted of a temporal clear corneal 2.75-mm main incision, through which ECP was performed using a curved 20-gauge endoscopic probe (Endo Optiks, Little Silver, NJ, USA) to deliver 810-nm laser (IRIDEX OcuLight SL., Mountain View, CA) under direct visualization from the base to the tip of each ciliary process until visible shrinkage and blanching occurred. Power settings were titrated $(100-250 \mathrm{~mW})$ as per the visualized response at a fixed, continuous pulse duration. All eyes received between 270 and $300^{\circ}$ of treatment using this technique. A cohesive ophthalmic viscoelastic device (Healon GVTM, Abbott Medical
Optics, USA) was injected into the ciliary space to facilitate access to the ciliary processes.

Where combined with cataract surgery, phacoemulsification (Stellaris, Bausch and Lomb, Surrey, UK) with in-the-bag IOL implantation of a monofocal three-piece implant (AMO Tecnis, Abbott Medical Optics, USA) was performed first, followed by ECP. All eyes received intracameral cefuroxime $(0.1 \mathrm{mg} / 0.1 \mathrm{ml})$ and subconjunctival dexamethasone $(3.3 \mathrm{mg} / 1 \mathrm{ml})$ at the end of surgery, and were prescribed a standard post-operative regime of topical dexamethasone and chloramphenicol.

\section{Trabeculectomy technique}

Trabeculectomy was performed as per the Moorfields Safer Surgery System [14] with a standard technique utilizing a corneal traction suture, fornix-based conjunctival incision, a $4 \mathrm{~mm} \times 3-\mathrm{mm}$ half-thickness scleral flap, mitomycin $\mathrm{C}$ (MMC) application for $3 \mathrm{~min}$, two pre-placed 10/0 monofilament nylon releasable flap sutures, Khaw Descemet's membrane punch (Duckworth and Kent Ltd), and a surgical peripheral iridectomy. A preservative-free post-operative regime consisting of topical dexamethasone every two hours, chloramphenicol four times a day, and atropine twice daily was prescribed.

\section{Results}

We performed either combined phaco-ECP or ECP alone in 62 eyes, of which $87.1 \%(54 / 62)$ had moderate or advanced progressive glaucoma and $12.9 \%$ (8/62) had early progressive glaucoma as defined by the Hodapp-Parrish-Anderson criteria [15]. Only $17.7 \%$ (11/62) of these eyes required subsequent trabeculectomy over a mean follow-up of $3.1 \pm 1.7$ years, whilst a further $1.6 \%(1 / 62)$ required subsequent Baerveldt Glaucoma Implant surgery. ECP therefore prevented the need for glaucoma filtration surgery in $80.6 \%$ (50/62) of cases.

Of those eyes that went on to have a subsequent trabeculectomy, two were excluded from analysis as they had less than 2-year post-operative data. The mean time from ECP to trabeculectomy in the remaining nine eyes was 22.7 (range 3.2-76.8) months with the majority (66.7\%) undergoing trabeculectomy $\geq 6$ months after ECP. A total of nine eyes matching the study group fulfilled all the criteria (as detailed in the "Methods" section) to be included in the control group. Controls consisted of eyes that underwent trabeculectomy without having undergone any prior glaucoma surgical procedures.

Table 1 shows baseline patient characteristics and mean visual acuity data in the study (group 1) and control 
Table 1 Baseline characteristics and visual acuity

\begin{tabular}{|c|c|c|c|}
\hline & $\begin{array}{l}\text { Group 1: prior ECP } \\
(n=9)\end{array}$ & $\begin{array}{l}\text { Group 2: controls } \\
(n=9)\end{array}$ & $p$-value \\
\hline Mean age (SD) & $77.8(7.7)$ & $69.9(6.7)$ & $0.015^{* a}$ \\
\hline Female:male & $6: 3$ & $7: 2$ & $0.598^{\mathrm{b}}$ \\
\hline Right eye:left eye & $4: 5$ & $4: 5$ & $1.000^{\mathrm{b}}$ \\
\hline Glaucoma diagnosis & & & $0.422^{\mathrm{b}}$ \\
\hline POAG, $n(\%)$ & $7(77.8)$ & $7(77.8)$ & \\
\hline NTG, $n(\%)$ & $1(11.1)$ & $2(22.2)$ & \\
\hline Uveitic glaucoma, $n(\%)$ & $1(11.1)$ & $0(0.0)$ & \\
\hline Lens status & & & $0.002 * \mathrm{c}$ \\
\hline Pseudophakic (PCIOL), $n(\%)$ & $9(100.0)$ & $2(22.2)$ & \\
\hline Phakic, $n(\%)$ & $0(0.0)$ & $7(77.8)$ & \\
\hline Prior laser treatment, $n(\%)$ & $5(55.6)$ & $5(55.6)$ & $0.214^{\mathrm{b}}$ \\
\hline ALT, $n(\%)$ & $4(44.4)$ & $1(11.1)$ & \\
\hline SLT, $n(\%)$ & $1(11.1)$ & $3(44.4)$ & \\
\hline None, $n(\%)$ & $4(44.4)$ & $5(44.4)$ & \\
\hline MMC dose & & & $0.628^{\mathrm{b}}$ \\
\hline $0.4 \mathrm{mg} / \mathrm{ml}, n(\%)$ & $4(44.4)$ & $3(33.3)$ & \\
\hline $0.2 \mathrm{mg} / \mathrm{ml}, n(\%)$ & $5(55.6)$ & $6(66.7)$ & \\
\hline Mean pre-op CDR (SD) & $0.91(0.05)$ & $0.84(0.10)$ & $0.061^{\mathrm{a}}$ \\
\hline Mean pre-op MD (SD) & $-20.9(9.2)$ & $-7.4(6.2)$ & $0.009^{* a}$ \\
\hline$\%$ eyes with $\mathrm{MD}>12 \mathrm{~dB}(\%)$ & $7(77.8)$ & $1(11.1)$ & $0.015^{* c}$ \\
\hline Mean pre-op VFI (SD) & $40.3(33.4)$ & $81.1(19.6)$ & $0.020 * \mathrm{a}$ \\
\hline \multicolumn{4}{|l|}{$\log$ MAR BCVA } \\
\hline Pre-op (SD) & $0.34(0.29)$ & $0.12(0.24)$ & $0.063^{\mathrm{a}}$ \\
\hline Pre-op BCVA $\geq 0.80, n(\%)$ & $3(33.3)$ & $0(0.0)$ & $0.029 * \mathrm{~b}$ \\
\hline 2 years post-op (SD) & $0.72(1.00)$ & $0.11(0.33)$ & $0.060^{\mathrm{a}}$ \\
\hline
\end{tabular}

$S D$, standard deviation; $E C P$, endoscopic cyclophotocoagulation; $P O A G$, primary open-angle glaucoma; $N T G$, normal tension glaucoma; $P C I O L$, posterior chamber intraocular lens; $A L T$, argon laser trabeculoplasty; $S L T$, selective laser trabeculoplasty; $C D R$, cup to disc ratio; $M D$, mean deviation; $V F I$, visual field index; $B C V A$, best-corrected visual acuity. ${ }^{a}$ Mann-Whitney $U$ test, ${ }^{b}$ Likelihood ratio chi-squared test, ${ }^{c}$ Fisher's exact test, *statistically significant (group 2) groups. There was no significant between-group difference in best-corrected visual acuity (BCVA) at 2 years. Mean follow-up was $4.4( \pm 1.9)$ and $4.4( \pm 1.8)$ years in groups 1 and 2, respectively. All eyes were followed up for at least 2 years, with $55.6 \%$ (5/9) and $66.7 \%$ (6/9) reaching 4 years' follow-up in groups 1 and 2, respectively.

Pre-trabeculectomy mean baseline IOPs were comparable at $23.7 \pm 7.7 \mathrm{mmHg}$ and $26.0 \pm 6.7 \mathrm{mmHg}(p=0.452)$ in groups 1 and 2 , respectively, on a similar mean of $3.4 \pm 0.9$ and $2.8 \pm 1.4$ anti-glaucoma medications for groups 1 and 2 , respectively ( $p=0.274)$. Groups 1 and 2 had $33.3 \%(3 / 9)$ and $22.2 \%(2 / 9)$ of eyes on oral acetazolamide, respectively $(p=0.599)$.

Figure 1 compares the change in IOP over time. At 2 years, the mean IOP was significantly and similarly reduced to $10.6 \pm 5.2 \mathrm{mmHg}$ and $12.9 \pm 4.0 \mathrm{mmHg}$ $(p=0.285)$, on a mean of $0.1 \pm 0.3$ and $0.1 \pm 0.4$ antiglaucoma medications $(p=0.931)$ for groups 1 and 2 , respectively. No eyes in either group were on oral acetazolamide after trabeculectomy surgery. Proportionately, more eyes had IOP $<10 \mathrm{mmHg}$ at final follow-up in group 1 (55.6\% vs. $22.2 \%$ ), but this difference did not reach statistical significance $(p=0.335)$.

At 2 years, complete success rates were $77.8 \%$ (7/9) and $88.9 \%(8 / 9)$ in groups 1 and 2 , respectively $(p=0.527)$. Qualified success rates were $11.1 \%(1 / 9)$ in both groups. Combined success rates were therefore $88.9 \%(8 / 9)$ and $100.0 \%$ (9/9) in groups 1 and 2, respectively (0.318). There was an $11.1 \%$ (1/9) failure rate in group 1, and no failures in group $2(p=0.318)$. Kaplan-Meier survival (Fig. 2) showed no statistically significant difference between the two groups using log rank, Breslow, or Tarone-Ware tests $(p=0.317)$.

Further interventions and post-operative complications in each group are compared in Table 2. Two eyes in group 1 and one in group 2 experienced transient numerical post-operative hypotony within the first week, 


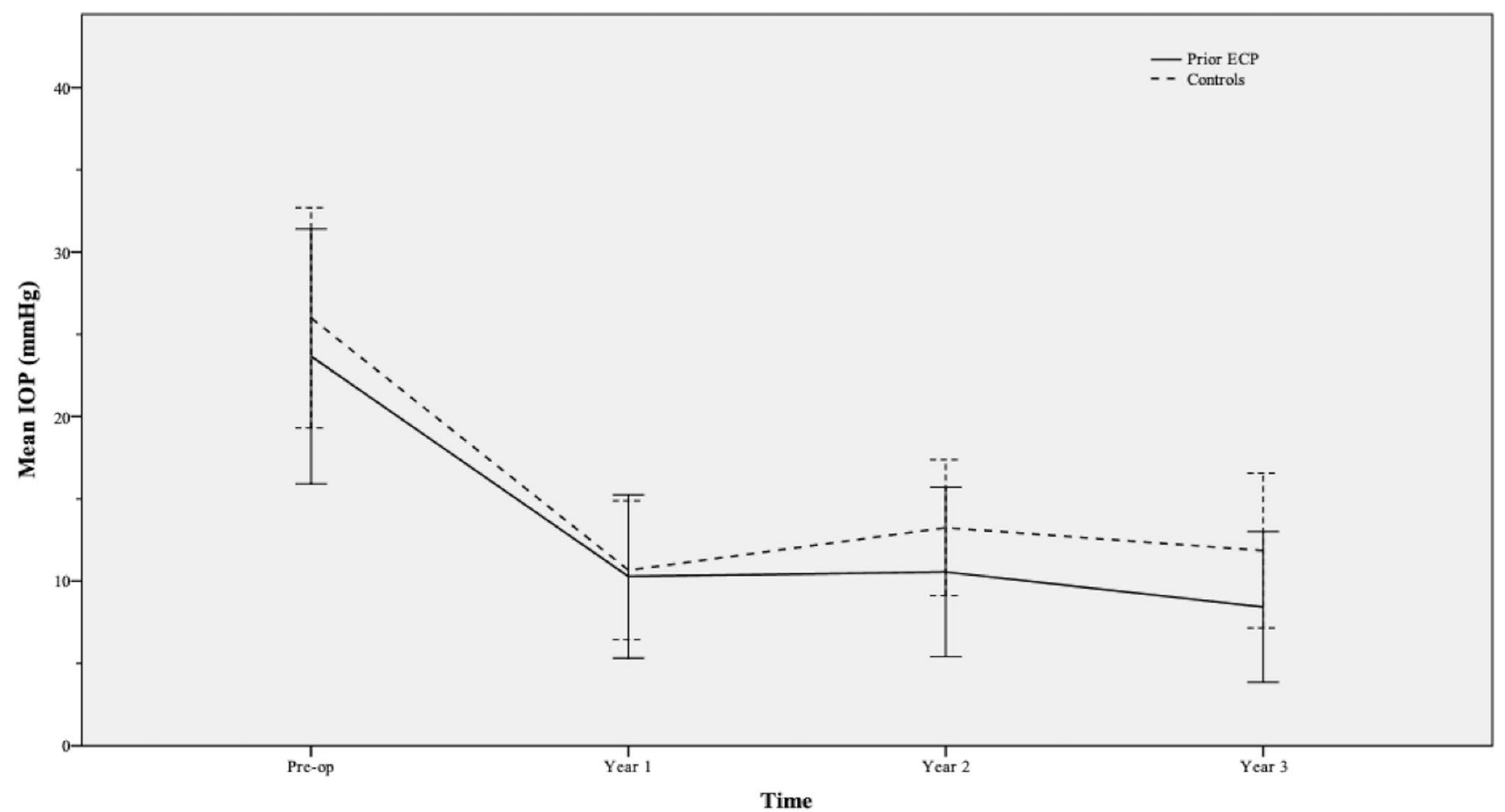

Fig. 1 Graph comparing post-operative intraocular pressure (IOP) after trabeculectomy surgery in each group. Error bars represent \pm 1 standard deviation

Fig. 2 Kaplan-Meier survival function with time. $\mathrm{ECP}=$ endoscopic cyclophotocoagulation

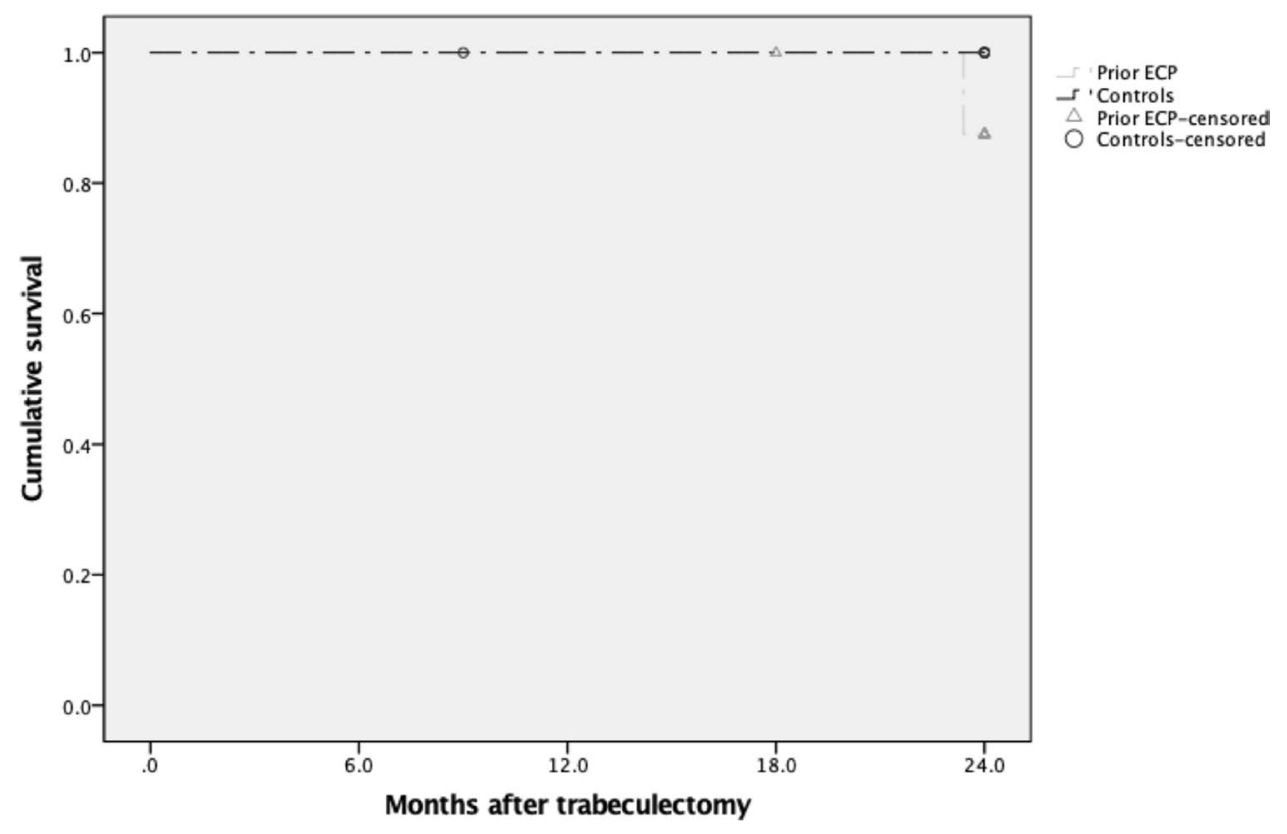

all resolving within the first month with conservative management consisting of either intracameral injection of Healon GV together with a course of oral prednisolone (both group 1 cases), or oral prednisolone alone (group 2 case). None of these eyes developed any hypotony-related complications at any time point. 
Table 2 Further interventions and post-operative complications after trabeculectomy

\begin{tabular}{llll}
\hline & $\begin{array}{l}\text { Group 1: Prior ECP } \\
(n=9)\end{array}$ & $\begin{array}{l}\text { Group 2: controls } \\
(n=9)\end{array}$ & $p$-value \\
\hline $\begin{array}{l}\text { Further interventions } \\
\text { Topical medications restarted }\end{array}$ & & & \\
$\quad \begin{array}{l}\text { d drop, } n(\%) \\
\geq 2 \text { drops, } n(\%)\end{array}$ & 0 & 1 (month 6) & $0.524^{\mathrm{b}}$ \\
BNR +5FU, $n$ (\%) & 1 & 0 & $0.248^{\mathrm{b}}$ \\
Phacoemulsification, $n(\%)$ & 0 & 3 & $0.029^{*}$ \\
Repeat trabeculectomy & 0 & 3 & \\
Baerveldt Glaucoma Implant & 0 & 0 & \\
Complications & & 1 (month 36) & \\
$\quad$ CMO & 1 (CRVO prior to ECP) & 0 & $0.229^{\mathrm{b}}$ \\
Hyphaema & 0 & 0 & \\
Persistent uveitis & 0 & 0 & $0.524^{\mathrm{b}}$ \\
Early hypotony (numerical) & 2 & 1 & \\
Hypotony (clinical) & 0 & 0 & $0.229^{\mathrm{b}}$ \\
Loss of light perception & 1 (month 23) & 0 & $0.229^{\mathrm{b}}$ \\
New CRVO & 1 (CRVO with CMO month 15) & 0 & \\
\hline
\end{tabular}

${ }^{\mathrm{b}}$ Likelihood ratio chi-squared test, *statistically significant test, Hypotony defined as IOP $<6 \mathrm{mmHg}$ using Goldmann Applanation Tonometry (GAT). BNR bleb needling revision, 5FU 5-fluorouracil, $C M O$ cystoid macular oedema, $C R V O$ central retinal vein occlusion

\section{Discussion}

This case-controlled study compares the long-term trabeculectomy outcomes in eyes that have previously undergone ECP with those in eyes not undergoing prior ECP. Concerns of a greater risk of chronic hypotony, inflammation, and bleb failure following a subsequent trabeculectomy mean that ECP, despite being a faster MIGS procedure, has not universally been adopted as a first-line option in moderate and advanced glaucoma. Trabeculectomy therefore has remained the firstline procedure of choice in this group of patients. Although several studies have previously reported the success of ECP and transscleral cyclophotocoagulation following failed GFS $[8,13]$, none have previously reported the efficacy and safety of trabeculectomy surgery following prior ECP. We demonstrate that prior ECP does not affect the long-term success of subsequent trabeculectomy surgery, and suggest that ECP can be safely offered as a first-line glaucoma procedure in eyes with good visual potential that have moderate or advanced progressive open-angle glaucoma.

We found no statistically significant difference between the study and control groups with regard to the primary and secondary outcomes. Trabeculectomy success rates were similar in both groups, although more eyes required anti-glaucoma medications for IOP control in the study group. We used a low-target IOP $(<16 \mathrm{mmHg})$ to define success due to the high proportion of eyes with advanced glaucoma in the study group, as evidenced by a mean visual field mean-deviation of $-20.9 \mathrm{~dB}$ (Table 1). Taking this into account, our overall trabeculectomy success rate of $88.9 \%(8 / 9)$ in the study group compares favourably with primary trabeculectomy success rates reported in the literature [16].

The outcomes of eyes undergoing ECP and not requiring subsequent GFS was beyond the scope of this study, given the strong existing evidence base. Various studies have previously reported the safety and efficacy of ECP in advanced POAG [8-10, 17] with one study reporting a significant IOP reduction from 23.1 to $12.1 \mathrm{mmHg}$ at 2 years [10]. Another study found a $94 \%$ 1-year and $82 \%$ 2-year success rates following ECP [8]. A third study reported a significant reduction in the medication burden following ECP [9]. Several studies have also found that combined ECP and phacoemulsification is significantly better at IOP and glaucoma medication reduction than phacoemulsification alone, with the beneficial effects of ECP still evident at 36 months and with comparable refractive outcomes [7, 18-20].

Another study investigating the outcomes of ECP following prior glaucoma drainage device implantation reported a mean IOP reduction from 24 to $15 \mathrm{mmHg}$ and a mean medication reduction from 3.2 to $1.5(p<0.001)$, with no serious complications [21]. Other studies have also shown a good safety profile for ECP, suggesting that any detrimental pro-inflammatory effects on a future trabeculectomy may be minimal. In addition, Chen et al. found no cases of hypotony or phthisis and a study by Lima et al. found cases of hypotony were limited to those eyes which had multiple previous operations or more complex pathology, with the hypotony risk also increasing with increasing number of clock hours of cyclophotocoagulation $[8,10]$. 
Although none of the eyes in our study group required further GFS following trabeculectomy, one eye with advanced uveitic glaucoma, poor baseline visual acuity, and retinal hypoperfusion at presentation required BNR at month 15 . This eye was the only eye deemed a failure in the study group due to loss of light perception at month 23 (Table 2). Two other eyes in the study group, neither of which experienced early post-operative hypotony, developed delayed persistent numerical hypotony after 6 months in the absence of clinical features of hypotony or its complications (shallow AC, choroidal detachment, macular folds, or reduced visual acuity). Both eyes were of the same patient, maintained good visual acuity, and did not require any further surgical intervention for the low IOP over a long follow-up of 4 years. Together with the fact that this was a highly myopic patient with thin central corneal thicknesses of 472 and 482 $\mu$, we attributed the numerical hypotony to a post-operative change in corneal biomechanical properties rather than the prior ECP treatment. Recent studies have demonstrated that Goldmann Applanation Tonometry (GAT) underestimates true IOP in eyes with GFS as a result of an increase in corneal hysteresis [22]. We did not have access to an Ocular Response Analyzer ${ }^{\circledR}$ (Reichert Inc., USA) in order to measure corneal hysteresis and corneal-compensated IOP, but were reassured by the absence of clinical features of hypotony in spite of the low GAT-measured IOPs.

Another two eyes in the study group developed cystoid macular oedema (CMO) post-operatively. However, this was associated with a new-onset retinal vein occlusion (RVO) at month 15 in one eye and recurrent $\mathrm{CMO}$ at year 4 in another eye following an RVO 9 months before the prior ECP. Both eyes maintained excellent IOP off topical medications throughout. There were no other cases of CMO, persistent fibrinous uveitis, or hyphaema to suggest that prior ECP conferred a greater risk of chronic inflammation after subsequent trabeculectomy.

Clinically, we found no evidence of heightened bleb encapsulation in eyes undergoing trabeculectomy after prior ECP. Although it is well known that bleb encapsulation is influenced by a number of factors including young age and prior incisional surgery [23], the effect of prior ECP on bleb encapsulation is not well researched. More eyes in our study group required long-term anti-glaucoma medications to achieve IOP control (Table 2), suggesting a stronger post-trabeculectomy encapsulation response in eyes with prior ECP. Conversely, however, fewer eyes in the study group required BNR compared to the control group. Of the three eyes requiring BNR in the control group, $66.7 \%$ still achieved complete success at 6 years. One of these eventually underwent Baerveldt Glaucoma Implant surgery at month 36 , but was still deemed a qualified success at month 24 .

It may be that low IOPs were still achieved in the study group despite the presence of some bleb encapsulation because aqueous production rates had been chronically lowered by the ECP. To objectively assess bleb encapsulation and failure rates after prior ECP, therefore, there is a need for future histological and prospective clinical studies. Given the small numbers involved, our study may have been underpowered to detect a real difference in bleb failure rates between the groups. Nevertheless, to the best of the authors' knowledge, this is the first study to address the effect of prior ECP on long-term trabeculectomy failure rates. Future randomized, controlled prospective studies in this area would be a valuable addition to the evidence base.

A limitation of this study was that, despite our attempts to closely match the groups, the study group had a significantly higher mean age, worse visual field mean-deviation, and worse pre-operative BCVA (Table 1) than the control group. This selection bias was hard to avoid as patients undergoing initial ECP were older patients (and therefore likely to have more advanced glaucoma) with multiple systemic comorbidities, where successful glaucoma control with a less invasive MIGS procedure was preferable to the peri-operative risks of a trabeculectomy and the need for frequent post-operative outpatient visits.

All the trabeculectomies in the study group were in pseudophakic eyes, whereas this was the case in only $22.2 \%$ in the control group (Table 1). This difference reflects the fact that in our unit all eyes in the study group underwent ECP either as a combined procedure with phacoemulsification or as a standalone if already pseudophakic. All eyes in the control group were open-angle glaucoma cases with visually asymptomatic cataracts and uncontrolled IOP where the lens was not felt to be a contributory factor. Trabeculectomy surgery was therefore performed as a standalone primary procedure in the control group.

In this study, subsequent GFS was required in only 12 of the 62 eyes (19.4\%) undergoing ECP over a 3-year period. All eyes undergoing ECP in our study would traditionally have undergone GFS as a primary surgery. ECP therefore led to an $80.6 \%$ reduction in trabeculectomy or glaucoma drainage device surgery. Although beyond the scope of our study, this would have had multiple cost implications with regard to reducing the number of post-operative visits as well as increasing theatre throughput given the reduced surgical time for ECP as compared to trabeculectomy.

However, caution should be taken when offering ECP in uveitic eyes or in Black and minority ethnic groups due to the greater risk of hypotony, prolonged inflammation, and chronic macular oedema [24]. Since the present study only included one patient of African-Caribbean ethnicity, our results cannot necessarily be applied to non-Caucasian populations. The main weakness of this study is the relatively small number of cases in the study group. However, it would take a long time to accumulate a substantially larger sample size with a long follow-up, given our low 3-year rates of subsequent filtration surgery following ECP. 
With the global COVID-19 pandemic resulting in a colossal backlog of glaucoma follow-ups, a large surge in the number of patients requiring vision-preserving glaucoma surgery is envisaged in the very near future. Trabeculectomy may not be the best option to cater for this imminent glaucoma workload in the current climate because of its intensive post-operative follow-up as well as longer surgical time. We believe glaucoma specialists will need to not only consider clinically safe and effective, but also time- and costeffective initial procedures as alternatives to trabeculectomy in order to adequately tackle the expected surge of advanced progressive glaucoma in the aftermath of the COVID-19 pandemic. Our results suggest that ECP is one such alternative, as it does not compromise the outcomes of a future trabeculectomy should it be required, whilst also reducing the need for GFS by up to $80 \%$ over a 3 -year period.

\section{Conclusions}

Our study demonstrates that ECP does not compromise the long-term safety or efficacy of future trabeculectomy surgery and should therefore be considered an initial minimally invasive procedure in progressive moderate and advanced glaucomas.

Code availability Not applicable.

\section{Declarations}

Ethics approval For this type of retrospective study, formal consent was not required. All procedures were performed as part of routine clinical care following informed patient consent and the study was carried out in accordance with the ethical standards of the local research committee and with the 1964 Declaration of Helsinki and its later amendments.

Conflict of interest The authors declare no competing interests.

\section{References}

1. Bloom PA, Tsai JC, Sharma K et al (1997) "Cyclodiode": transscleral diode laser cyclophotocoagulation in the treatment of advanced refractory glaucoma. Ophthalmology 104(9):1508-19 (discussion 1519-20)

2. Singh K, Dangda S, Ahir N et al (2017) Diode laser cyclophotocoagulation paves way to a safer trabeculectomy in eyes with medically uncontrolled intraocular pressure. Int Ophthalmol 37(2):365-370

3. Falkenberry SM, Siegfried CJ (2009) Endocyclophotocoagulation. Middle East Afr J Ophthalmol 16(3):130-133

4. Shields MB (1986) Intraocular cyclophotocoagulation. Trans Ophthalmol Soc U K 105:237-241

5. Seibold LK, SooHoo JR, Kahook MY (2015) Endoscopic cyclophotocoagulation. Middle East Afr J Ophthalmol 22(1):18-24
6. Rathi S, Radcliffe NM (2017) Combined endocyclophotocoagulation and phacoemulsification in the management of moderate glaucoma. Surv Ophthalmol 62(5):712-715

7. Pérez Bartolomé F, Rodrigues IA, Goyal S et al (2018) Phacoemulsification plus endoscopic cyclophotocoagulation versus phacoemulsification alone in primary open-angle glaucoma. Eur J Ophthalmol 28(2):168-174

8. Chen J, Cohn RA, Lin SC et al (1997) Endoscopic photocoagulation of the ciliary body for treatment of refractory glaucomas. Am J Ophthalmol 124(6):787-796

9. Morales J, Al Qahtani M, Khandekar R et al (2015) Intraocular pressure following phacoemulsification and endoscopic cyclophotocoagulation for advanced glaucoma: 1-year outcomes. J Glaucoma 24(6):157-162

10. Lima FE, Carvalho DM, Avila MP (2010) Phacoemulsification and endoscopic cyclophotocoagulation as primary surgical procedure in coexisting cataract and glaucoma. Arq Bras Oftalmol 73(5):419-422

11. Cohen A, Wong SH, Patel S et al (2017) Endoscopic cyclophotocoagulation for the treatment of glaucoma. Surv Ophthalmol 62(3):357-365

12. Ishida, (2013) Update on results and complications of cyclophotocoagulation. Curr Opin Ophthalmol 24(2):102-110

13. Lin SC (2008) Endoscopic and transscleral cyclophotocoagulation for the treatment of refractory glaucoma. J Glaucoma 17(3):238-247

14. Khaw PT, Chiang M, Shah P et al (2017) Enhanced trabeculectomy: the Moorfields safer surgery system. Dev Ophthalmol 59:15-35

15. Anderson RD, Patella VM (1999) Automated static perimetry, 2nd edn. Mosby, St. Louis

16. Kirwan JF, Lockwood AJ, Shah P et al (2013) Trabeculectomy in the 21st century: a multicenter analysis. Ophthalmology 120(12):2532-2539

17. Kaplowitz K, Kuei A, Klenofsky B et al (2015) The use of endoscopic cyclophotocoagulation for moderate to advanced glaucoma. Acta Ophthalmol 93(5):395-401

18. Francis BA, Berke SJ, Dustin L et al (2014) Endoscopic cyclophotocoagulation combined with phacoemulsification versus phacoemulsification alone in medically controlled glaucoma. J Cataract Refract Surg 40(8):1313-1321

19. Kang S, Luk S, Han H et al (2017) Refractive outcome of combined phacoemulsification and endoscopic cyclophotocoagulation. Int Ophthalmol 37(6):1311-1317

20. Siegel MJ, Boling WS, Faridi OS et al (2015) Combined endoscopic cyclophotocoagulation and phacoemulsification versus phacoemulsification alone in the treatment of mild to moderate glaucoma. Clin Exp Ophthalmol 43(6):531-539

21. Francis BA, Kawji AS, Vo NT et al (2011) Endoscopic cyclophotocoagulation (ECP) in the management of uncontrolled glaucoma with prior aqueous tube shunt. J Glaucoma 20(8):523-527

22. Pakravan M, Afroozifar M, Yazdani S (2014) Corneal biomechanical changes following trabeculectomy, phaco-trabeculectomy, Ahmed glaucoma valve implantation and phacoemulsification. J Ophthalmic Vis Res 9(1):7-13

23. Richter CU, Shingleton BJ, Bellows AR et al (1988) The development of encapsulated filtering blebs. Ophthalmology 95(9):1163-1168

24. Edmiston AM, SooHoo JR, Seibold LK et al (2018) Postoperative inflammation after endoscopic cyclophotocoagulation: racial distribution and effect on outcomes. J Glaucoma 27(3):266-268

Publisher's note Springer Nature remains neutral with regard to jurisdictional claims in published maps and institutional affiliations. 